\title{
Prácticas inclusivas de los docentes
}

\section{Teacher-inclusive practices}

\author{
Wilson García Guevara ${ }^{1 *}$, Óscar Belesaca ${ }^{2}$ y Gabriela Jara Saldaña ${ }^{3}$ \\ ${ }^{1}$ Universidad Católica de Cuenca \\ 2 Unidad Educativa Juan Bautista Stielhe, Cuenca \\ 3 Proyecto de vinculación con la sociedad UDIPSAI \\ *wgarciag@ucacue.edu.ec
}

DOI: https://doi.org/10.26871/killkana_social.v2i2.303

\begin{abstract}
Resumen
El presente estudio tiene como objeto determinar el nivel de aplicación de prácticas inclusivas por parte de docentes y estudiantes en la Unidad Educativa Juan Bautista Stielhe de la ciudad de Cuenca en el año lectivo 2015-2016. Las prácticas inclusivas, reflejan la cultura y las políticas inclusivas, tienen que ver con asegurar que las actividades de aprendizaje en el aula y las extraescolares promuevan la intervención de todos los dicentes y que consideren el conocimiento y la experiencia adquiridos por ellos fuera de la institución educativa; la enseñanza y los apoyos se integran para orquestar el aprendizaje y superar las barreras al aprendizaje y la participación; el personal docente y administrativo moviliza recursos de las instituciones y de la comunidad para mantener el aprendizaje activo de todos. Para el desarrollo de este trabajo de investigación se basó en el método cualicuantitativo de tipo descriptivo y exploratorio, con una población de 19 docentes y 118 estudiantes de los niveles de básica superior y bachillerato de la institución; para la recolección de la información, se aplicó parte del Índice de Inclusión de la UNESCO, adaptación de Rosa Blanco, que consta de veinte indicadores de inclusión permitiendo abstraer información de los docentes y estudiantado. La información es procesada estadísticamente, se analizan los resultados y se concluye que los docentes presentan una predisposición y prácticas favorables a la inclusión cumpliendo con sus obligaciones curriculares, hacen un mayor uso de las estrategias de organización y manejo efectivo del aula, en los procesos de aprendizaje, mientras que los estudiantes tienen una buena percepción y predisponían a la inclusión educativa, las autoridades brindan las facilidades para cumplir el proceso inclusivo cumpliendo con las leyes y normativas del país.
\end{abstract}

Palabras clave: adaptaciones curriculares, inclusión educativa, necesidades educativas especiales, prácticas inclusivas.

\begin{abstract}
The objective of this study is to determine the level of application of inclusive practices by teachers and students in the Juan Bautista Stielhe Educational Unit of the city of Cuenca in the 2015-2016 school year. Inclusive practices, reflecting inclusive culture and policies, have to do with ensuring that classroom and after-school learning activities promote the intervention of all children and that they consider the knowledge and experience acquired by them outside the institution. educational teaching and support are integrated to orchestrate learning and overcome barriers to learning and participation; the teaching and administrative staff mobilizes resources from the institutions and the community to maintain active learning for all. For the development of this research work, it was based on the descriptive and exploratory qualitative and quantitative method, with a population of 19 teachers and 118 students of the upper and upper secondary levels of the institution; for the collection of information, part of the Inclusion Index of UNESCO was applied, adapted from Rosa Blanco, which consists of twenty inclusion indicators allowing to abstract information from teachers and students. The information is processed statistically, the results are analyzed and it is concluded that the teachers have a predisposition and favorable practices to the inclusion fulfilling their curricular obligations, make a greater use of the strategies of organization and effective management of the classroom, in the processes of learning, while students have a good perception and predisposed to educational inclusion, the authorities provide the facilities to comply with the inclusive process complying with the laws and regulations of the country.
\end{abstract}

Key words: curricular adaptations, inclusive education, special educative needs, inclusive practices.

\section{Introducción}

En la Unidad Educativa Juan Bautista Stiehle ubicada en el cantón Cuenca de la provincia del Azuay, surge la necesidad de investigar el nivel de prácticas inclusivas por parte de los docentes debido a que el psicólogo del Departamento de Consejería (DECE) reporta mediante un oficio que existe una resistencia a recibir a estudiantes sujetos de inclusión educativa, a causa de la falta de adaptaciones 
curriculares, desconocimiento de la aplicación de estrategias y recursos pedagógicos, así como el reclamo continuo de los docentes a la autoridad institucional de un proyecto de tutoría adicional para cada niño con discapacidad que atiendan las necesidades diversas de los mismos. Frente a esta situación se presentan las siguientes interrogantes: ¿Cuáles son los niveles de buenas prácticas ante la inclusión educativa? ¿El docente está capacitado para enfrentar el reto de la inclusión? ¿Se practica una verdadera inclusión educativa por parte de los docentes? Para dar respuestas a estas interrogantes se aplicó parte del índice de inclusión de la UNESCO, adaptación de Rosa Blanco para Latinoamérica, el mismo que fue aplicado a docentes y estudiantes de la Unidad Educativa ya mencionada. En el marco conceptual se abarcan los conceptos de inclusión educativa como en el informe Warnock (1978) citado por (Montero, 2016), manifiesta que la inclusión localiza su atención en el apoyo fijo que necesita una persona en función de sus diferentes necesidades que pueden ser permanentes o transitorias, actitudes y percepciones de los docentes hacia este campo de estudio. También se abarca estadísticas de la UNESCO a nivel de Latinoamérica, en relación al Ecuador se fundamentan estadísticas en artículo de la Universidad Casa Grande de Guayaquil.

Se basa en el marco legal del Ecuador fundamentado en la Constitución de la República, en la Ley Orgánica de Educación Intercultural "LOEI”, acuerdos ministeriales y acuerdos internacionales que consideran a la inclusión educativa como un derecho humano y ciudadano. Es fundamental que en el sistema educativo ecuatoriano se implemente una política educativa en las instituciones educativas para la aplicación de las adaptaciones curriculares que "son adecuaciones, modificaciones o ajustes que se realizan a los diferentes elementos del currículo, transformándolo, flexibilizando y facilitando el proceso de enseñanza aprendizaje de los niños, niñas y/o adolecentes con necesidades educativas especiales" (Vicepresidencia del Ecuador, 2011, p.13).

Desde el punto de vista pedagógico se pudo conocer cómo aplican los docentes y el estudiantado la inclusión educativa, con estos resultados las autoridades educativas podrán plantear las acciones que se orientan a transformar el estado actual del problema detectado, y dar respuesta a las necesidades de este grupo de estudio, brindando una actualización de los niveles de prácticas inclusivas por parte de los docentes y la asimilación que tienen los estudiantes de esta forma de educación. Pedagogos actuales plantean la necesidad de formar a los futuros docentes en una cultura de la diversidad que fundamente crítica y reflexivamente la educación inclusiva. Partiendo de esta concepción es necesario precisar que la educación actual es activa, fundamentada por destrezas y no por conocimientos (López, 2012).

Desde el punto de vista social, este trabajo permite atender parte de las necesidades más importantes que vive la educación actual dentro del ámbito inclusivo. Si los docentes no realizan buenas prácticas inclusivas, resultarían afectados los estudiantes, que no pueden ser tratados de acuerdo a sus características individuales.

A más de los beneficios que se presentan para los estudiantes con necesidades educativas especiales (NEE), el estudiantado en general y los docentes, también se beneficiarán a sus representantes legales, debido a que evitarán la incertidumbre del presente y futuro de su representado.

La conclusión que se llegó en este trabajo de investigación es que los docentes tienen un buen nivel de aplicación de prácticas inclusivas porque la planificación y el desarrollo de las clases responden a la diversidad del estudiantado, las clases se hacen accesibles a todos los dicentes, y promueven la comprensión de las diferencias individuales.

\section{Marco teórico}

En la actualidad uno de los problemas que enfrenta la educación en el mundo es la dificultad y el obstáculo que presentan los docentes para realizar la inclusión educativa en el aula. Según datos de la UNESCO en América Latina y el Caribe, solo entre el $20 \%$ y el $30 \%$ de los niños/as con necesidades educativas especiales asisten a la escuela y que estos cuando entran, suelen ser excluidos enseguida de los sistemas educativos. Según la UNESCO el acceso a la educación cambia por el tipo y el grado de discapacidad, y la mayor parte de matriculados se concentran en la educación primaria; en el Ecuador aproximadamente el $10 \%$ termina el nivel secundario (Crosso, 2010, p.80).

La Ley Orgánica de Educación Intercultural en el artículo 47 establece: "El Estado ecuatoriano garantizará la inclusión e integración de las personas con discapacidad..." y en el artículo 128 establece: "son estudiantes con necesidades educativas especiales aquellos que requieren adaptación temporal o permanente" (Ministerio de Educación, 2011, p.24), el Acuerdo $\mathrm{N}^{\circ}$ 295-13 del Ministerio de Educación (2013), define a la inclusión educativa como el "proceso de identificar y responder a la variedad de necesidades especiales de todo el estudiantado a través de la mayor participación en el aprendizaje, las culturas y las comunidades, a fin de reducir la exclusión en la educación..."(p.5)., por lo tanto, las instituciones educativas, así como sus actores deberán velar por el cumplimiento de las mismas y más aún el docente como facilitador del aprendizaje que mediante buenas prácticas inclusivas, demostrará y evidenciará su labor docente cumpliendo con el marco legal vigente.

Reconociendo los fundamentales cambios existentes por una globalización y una colectividad del conocimiento, uno de los retos más difíciles para el sistema educativo es entender, y dar a conocer políticas y prácticas para la inclusión que permitan conseguir los aprendizajes elementales de la educación obligatoria a todo el estudiantado (Martínez, 2011). El profesorado deberá capacitarse en todo lo necesario para apoyar al niño o niña que requiere de un 
proceso de inclusión permitiéndole un desarrollo de igual calidad que el resto de sus compañeros.

La buena práctica inclusiva se considera "toda actuación que se oriente, a partir del compromiso del profesorado, alumnado y familias, a promover la presencia, la participación y el éxito de todo el alumnado, sobre todo de aquellos en mayor situación de vulnerabilidad" (Durán, Marchesi, y Hernández Izquierdo, 2009, p.6). Las prácticas inclusivas es el hecho de hacer operativa y poner en marcha la inclusión educativa de las personas en el ámbito escolar, sin perjurios sociales ya sea por discapacidad o porque son de diferentes orígenes.

La inclusión educativa pretende reconocer y responder a la variedad de las necesidades de todo el estudiantado a través de una mejor participación y cooperación en el aprendizaje (UNESCO, 2016).

El Informe Warnock (1978) citado por (Montero, 2016), manifiesta que la inclusión focaliza su atención en el apoyo fijo que necesita una persona en función de sus diferentes necesidades que pueden ser permanentes o transitorias. Por lo tanto la institución educativa será la responsable de atender holísticamente las necesidades del educando a partir de la destrucción de toda barrera existente que imposibilite su aprendizaje y a su vez brindando las facilidades que le permitan potencializar todas sus capacidades y aptitudes para el desarrollo personal.

El objeto de la inclusión es fomentar los valores como la solidaridad, respeto a las diferencias individuales, tolerancia para obtener una convivencia armónica y la práctica fundamentada en el diálogo y de esa manera resolver conflictos. También eliminan las barreras del aprendizaje que estén relacionadas con la infraestructura de la institución, sistema de comunicación interpersonal o colectiva, recursos didácticos, currículos, contexto geográfico y cultural; y se forman ciudadanos autosuficientes, independientes, capaces de desenvolverse activa y participativamente en el ámbito social con unas buenas bases de relaciones intrapersonales para fundamentar su éxito en el campo laboral y social.

La práctica de una educación inclusiva debe garantizar el acceso para todos a un sistema general educativo, una buena adaptación, aceptación y el buen trato de los estudiantes con necesidades educativas especiales, no solo debería centrar en las actitudes que tienen los docentes a la inclusión sino que deberían tener buenas prácticas de inclusión, de que serviría si un docente tiene una buena actitud a la inclusión y no tienen los conocimientos teóricos y prácticos necesarios para realizar una inclusión acorde a las necesidades del educando. De esta manera, transformando el paradigma de inclusión, los estudiantes que tienen necesidades educativas especiales, se benefician de los procesos de inclusión, y además toda la comunidad educativa, ya que las prácticas inclusivas son consideradas como un estándar de calidad que brinda el servicio educativo de la institución.

La Ley Orgánica de Educación Intercultural en el artículo 2 literal "v" plantea "la equidad e inclusión aseguran a todas las personas el acceso, permanencia y culminación en el Sistema Educativo. Garantiza la igualdad de oportunidades a comunidades, nacionalidades y grupos con necesidades educativas especiales y desarrolla una ética de la inclusión..." (Ministerio de Educación, 2011, p.10).

La educación se centraría en el ser humano y aseguraría su desarrollo holístico, el docente tiene un papel fundamental en el campo de la inclusión de los estudiantes por lo que tiene una incuestionable y absoluta responsabilidad ante los grupos que atiende durante el proceso de enseñanza-aprendizaje (Garnique, 2016, p.60). Los profesionales de la educación deberían estar a la vanguardia en prácticas y métodos inclusivos en la institución educativa; si no se tiene prácticas inclusivas, inconscientemente excluirían a los estudiantes. Cabe recordar que la inclusión no solo incluye a los niños y niñas con discapacidad, sino que debe reconocerse como la preocupación por un aprendizaje y un rendimiento escolar que llegue a los estándares de calidad de todo el estudiantado que propone el Ministerio de Educación.

Según la programación neurolingüística todos aprenden de diferentes maneras, cada estudiante tiende a desarrollar diferentes habilidades al momento de adquirir alguna destreza, y cada docente el método que le resulte más apropiado para enseñar. Algunos profesores eligen los medios audiovisuales para dictar sus clases, otros lo hacen de forma oral y otros prefieren enseñar mientras los alumnos trabajan manualmente, por ejemplo, dentro de los laboratorios (Tocci, 2013). Todas estas formas de enseñanza-aprendizaje se fundamentarían en prácticas de inclusión, debido a que todos los dicentes no aprenden, así por ejemplo, un estudiante que tenga una deficiencia visual tomaría poca atención al pizarrón y prestaría más atención a las instrucciones del docente de forma oral.

Considerando que la inclusión educativa es fundamental en los sistemas de educación actual en Ecuador, para hacer efectiva esta política educativa es necesario que en las instituciones educativas se implementen adaptaciones curriculares que "son adecuaciones, modificaciones o ajustes que se realizan a los diferentes elementos del currículo, transformándolo, flexibilizando y facilitando el proceso de enseñanza aprendizaje de los niños, niñas y/o adolecentes con necesidades educativas especiales" (Vicepresidencia del Ecuador, 2011). Las adaptaciones curriculares se clasifican en dos tipos: a los elementos de acceso y al currículo propiamente dicho.

Por elementos de acceso se hace referencia a la infraestructura, como por ejemplo, la señalización, baterías sanitarias; a la comunicación, entre otros.

Para los elementos del currículo aquellos que se dividen: en adaptaciones curriculares significativas y adaptaciones curriculares menos significativas. Las adaptaciones curriculares significativas constituyen las permanentes, que están asociadas a la discapacidad, pudiendo ser la intelectual, física motora, auditiva, visual o mental, trastornos generalizados del desarrollo, en estos casos se necesitan 
adaptaciones curriculares a todos los elementos del currículo. Las adaptaciones curriculares menos significativas se relacionan con las necesidades educativas especiales no asociadas a la discapacidad que a la vez pueden necesitar de adaptaciones transitorias a determinado elemento del currículo; como en el caso de estudiantes con dotación superior, dificultades específicas de aprendizaje como la dislexia, discalculia, entre otras. (Ministerio de Educación, 2011).

Las Adaptaciones curriculares de acuerdo al grado de significatividad, según Burgos B. (2010), se pueden clasificar en tres categorías:

- Grado 1 (menos significativas) son modificaciones a los elementos de acceso al currículo como son la infraestructura, los apoyos personales, la organización institucional, los recursos y ayudas materiales, la organización en la escuela y en el aula entre otros.

- Grado 2 (significativas) son modificaciones a las estrategias metodológicas, las actividades, los recursos didácticos, la temporalización, entre otros.

- Grado 3 (muy significativas) se modifican los elementos básicos del currículo como contenidos, conocimientos, objetivos, resultados de aprendizaje, destrezas con criterio de desempeño, los criterios de evaluación y por tanto se modifican también las actividades, los recursos didácticos, las estrategias metodológicas.

En base a los resultados en la evaluación psicopedagógica, es el docente el encargado de realizar la adaptación que requiere el estudiante, para que se sienta parte y participe activamente del proceso educativo. Estas adaptaciones se realizan comenzando desde las menos significativas, grado 1, para luego considerar las significativas grado 2 y por último las grado 3 que son muy significativas que implican modificaciones de objetivos y/o contenidos, que repercute en el perfil de egreso del estudiante.

\section{Método}

Para el desarrollo de la investigación se basó en el método cualicuantitativo de tipo descriptivo y exploratoria en la cual se hace presente una población de 19 docentes y 118 estudiantes de los niveles de básica superior y bachillerato de la Unidad Educativa Juan Bautista Stielhe; para la recolección de la información se utilizó la técnica de la encuesta y como instrumento el cuestionario, que fue estructurado tomando como base parte del índice de inclusión de la UNESCO, adaptación de Rosa Blanco, que constó de 20 indicadores de inclusión permitiendo abstraer información de los docentes y estudiantado; para corroborar la información obtenida de las encuestas aplicadas se utilizó una ficha de observación, mediante los resultados de las encuestas se fundamenta el nivel de prácticas que tienen los docentes en inclusión educativa.

Una vez obtenida la información a través de la aplicación del instrumento se procesó la información mediante la tabulación, construcción de tablas y gráficos estadísticos en el programa Microsoft Excel; la interpretación y análisis de resultados, se realizó mediante el contraste de la investigación bibliográfica y los resultados obtenidos, los datos de los docentes con los datos de los estudiantes en base a los cuales se sustenta las conclusiones.

\section{Resultados}

Del análisis de los resultados de los docentes se observa que el 73,7\% está completamente de acuerdo en que realizan planificaciones y adaptaciones curriculares; con respecto a las adaptaciones a las evaluaciones el 84,2\% están completamente de acuerdo; el $57,9 \%$ se manifestó completamente de acuerdo en cuanto si las clases son accesibles para todos los estudiantes; el 89,5\% se encuentra completamente de acuerdo en cuanto si el profesorado se preocupan en ayudar el aprendizaje y la participación de todo el estudiantado; el 63,2\% están completamente de acuerdo en relación si las evaluaciones estimulan los logros de los estudiantes; el 89,5\% se encuentran completamente de acuerdo en cuanto a si la disciplina en el aula se basa en el respeto mutuo; el 89,5\% hace referencia al indicador completamente de acuerdo en cuanto si a los estudiantes se les forma para valorar a las personas de orígenes distintos o que se encuentren en una situación de discapacidad; el 78,9\% se encuentra completamente de acuerdo en relación a si los docentes utilizan la diversidad del alumnado como estrategia para desarrollar el proceso de enseñanza-aprendizaje; 47,4\% están parcialmente en desacuerdo en cuanto si el DECE realiza un trabajo con los estudiantes para facilitar el aprendizaje y la participación de los estudiantes; $47,4 \%$ se encuentra completamente de acuerdo en cuanto si los deberes enviados a la casa contribuyen al aprendizaje de todo el estudiantado; el 57,9\% se manifiesta completamente de acuerdo en cuanto si los recursos de la unidad educativa se distribuyen de una forma equitativa para realizar inclusión; y el 47,4\% se encuentra parcialmente en desacuerdo con respecto a si conoce y se aprovecha los recursos de la comunidad educativa para apoyar la inclusión.

Con relación a los estudiantes se obtuvieron los siguientes resultados: el 66,9\% se encuentra completamente de acuerdo, en si el docente utiliza diferentes técnicas con estudiantes que necesitan apoyo académico; el 56,8\% se encuentra completamente de acuerdo en si el docente realiza una adaptación a la evaluación para algún estudiante; el $66,9 \%$ se inclina por el indicador completamente de acuerdo en cuanto a la confianza que tiene el estudiante hacia el docente; el $63,6 \%$ se encuentra completamente de acuerdo en si los docentes están siendo parte del proceso enseñanzaaprendizaje mediante las opiniones y argumentación de ideas; $48,3 \%$ se encuentra completamente de acuerdo en cuanto a la equidad que tienen los docentes hacia el estudiantado; el 51,7\% se encuentra completamente de acuerdo en relación a la confianza y equidad que brinda el docente hacia el estudiantado dentro de las aulas de clases; el 82,2 \% se encuentra completamente de acuerdo que hace referencia a la aceptación e integración de estudiantes de diferentes 
orígenes y características intelectuales o físicas; el 77,1\% se refiere al indicador completamente de acuerdo en cuanto que los estudiantes mejoran su aprendizaje con la ayuda de un docente de apoyo; el 50,8\% fundamenta que esta completamente de acuerdo en cuanto a la comprensión que tiene el estudiante de las tareas enviadas a casa; el $63,6 \%$ se encuentra completamente de acuerdo en cuanto al cumplimiento de las tareas en casa; el 47,5\% se encuentra completamente de acuerdo en cuanto a que los dicentes tienen confianza a que los trabajos serán expuestos en las carteleras de la unidad educativa; y el $66,9 \%$ se encuentra completamente de acuerdo en cuanto si el docente utiliza recursos diferentes con estudiantes que necesitan apoyo académico.

Referente a los resultados de la ficha de observación aplicada se obtiene que un $61 \%$ de docentes evidencian en sus planificaciones las adaptaciones curriculares; un $63 \%$ de estudiantes tienen confianza en los docentes; un $53 \%$ de docentes tratan con equidad hacia los estudiantes, un $72 \%$ valora la diversidad de las personas, y un $42 \%$ se observa que la institución educativa apoya a la inclusión.

Existe una concordancia entre las respuestas de los docentes y estudiantes, como se observa en la tabla 1

Tabla 1

\begin{tabular}{lccc}
\hline Indicadores & Docente \% & Estudiante \% & Observación \% \\
\hline $\begin{array}{l}\text { Planificación y documen- } \\
\text { tos curriculares }\end{array}$ & 84,2 & 66,9 & 61 \\
$\begin{array}{l}\text { Confianza del estudiante } \\
\text { hacia el docente }\end{array}$ & 89,5 & 66,9 & 63 \\
$\begin{array}{l}\text { La equidad del docente } \\
\text { hacia el estudiantado }\end{array}$ & 89,5 & 51,7 & 53 \\
$\begin{array}{l}\text { Valorar la diversidad de } \\
\text { las personas }\end{array}$ & 89,5 & 82,2 & 72 \\
$\begin{array}{l}\text { Apoyo institucional a la } \\
\text { inclusión educativa }\end{array}$ & 57,9 & 66,9 & 42 \\
\hline
\end{tabular}

Fuente: Encuesta a docentes y estudiantes de la Unidad Juan Batista Stielhe.

\section{Conclusiones}

Luego del análisis e interpretación de los resultados obtenidos de las encuestas a docentes y estudiantes y de la ficha de observación, se concluye que los docentes tienen un buen nivel de aplicación de prácticas inclusivas por que la planificación y el desarrollo de las clases responden a la diversidad del estudiantado, las clases se hacen accesibles a todo los dicentes, las clases promueven la comprensión de las diferencias individuales, se implica activamente al estudiantado en su propio aprendizaje, los estudiantes aprenden de manera cooperativa, la evaluación estimula los logros de todo el estudiantado, se forma a los discente en un ambiente inclusivo para valorar a las personas que tienen un origen distinto al propio o que se encuentra en una situación de discapacidad, el profesorado se preocupa de apoyar el aprendizaje y la participación de todos, en su plan de clases por destreza realiza las adaptaciones curriculares y todo el estudiantado participa en actividades complementarias y extraescolares.
Los estudiantes tienen una percepción positiva de la inclusión educativa en la institución dando un mayor reconocimiento a la formación en valores hacia la diversidad individual ya sea de personas de otros orígenes o que tengan alguna discapacidad, reconociendo que tiene confianza al docente, valorando el trabajo cooperativo que tiene en su labor estudiantil; pero reconocen que la equidad que brinda el docente es uno de los puntos inconsistentes que se debe tratar, para mejorar las prácticas inclusivas en la institución; por lo tanto el estudiantado está cumpliendo un rol fundamental en el proceso inclusivo de la institución.

Las autoridades y personal administrativo cumplen una labor importante dando las pautas y las directrices para lograr la educación inclusiva; pero es el indicador con menos aceptación por parte de los docentes, estudiantes, lo que se corrobora con la ficha de observación, por lo que constituye aspecto a fortalecer en lo referente a las prácticas inclusivas en la institución.

Fundamentando en el análisis e interpretación de los resultados de la investigación, el nivel de aplicación de prácticas inclusivas es bueno, refutando al supuesto que se manifiesta en el oficio, entregado por el psicólogo del DECE de la Unidad Educativa Juan Bautista Stielhe, en donde manifiesta que existe una "resistencia a recibir a estudiantes sujetos de inclusión educativa, a causa de la falta de adaptaciones curriculares, desconocimiento de la aplicación de estrategias y recursos pedagógicos". Lo que constituyó en el impulso para el planteamiento del problema con la finalidad de investigar y mejorar el nivel de aplicación prácticas inclusivas en dicha institución educativa.

Los resultados obtenidos son exclusivos de la institución educativa Juan Bautista Stielhe; y no se pueden generalizar debido a que tiene una realidad educativa, social y económica propia.

Se concluye que en la Unidad Educativa Juan Bautista Stielhe existe un buen nivel de aplicación de prácticas inclusivas por parte de los docentes, estudiantado y a nivel de autoridades; los docentes tienen una buena predisposición, actitud y prácticas inclusivas, cumpliendo con sus obligaciones curriculares, formando a los discentes en un ámbito inclusivo y sin perjuicios; cumpliendo con las normativas que pide el Ministerio de Educación.

\section{Referencias Bibliográficas}

Crosso, C. (2010). El Derecho a la Educación de Personas con Discapacidad: impulsando el concepto de Educación Inclusiva. Revista Latinoamericana de educacion inclusiva.

Durán, D., Marchesi, 1., y Hernández Izquierdo, L. (2009). Guia para la reflexión y valoración de prácticas inclusivas. Madrid: Organización de Estados Iberoamericanos para la Educación, la Ciencia y la Cultura.

Garnique, F. (2016, enero). Perfiles educativos vol.34 no.137 México ene. 2012. 
López, N. (2012). Equidad educativa y diversidad. Buenos Aires: Copyright (C) UNESCO 2012.

Martínez, B. (2011). Luces y sombras de las medidas de atención a la inclusión educativa. Revista Interuniversitaria de Formación del Profesorado, 166.

Ministerio de Educación. (2011). Articulo 47. En LOEI (p. 24). Quito: Editora Nacional.

Montero, L. A. A. (2016, enero). El informe Warnock.

Tocci, A. (2013). Estilo de aprendizaje de los alumnos de ingeniería. Revista estilos de aprendizaje, 20.

UNESCO. (2016, enero). www.inclusioninternocional.org.

Vicepresidencia del Ecuador. (2011). Educación inclusiva y especial. Quito, Ecuador: Editorial Ecuador. 